\title{
Revitalising Pedrick's Approach to Reproducing Kernel Hilbert Spaces
}

\author{
Franciszek Hugon Szafraniec ${ }^{1}$
}

Received: 7 July 2020 / Accepted: 13 February 2021 / Published online: 26 April 2021

(c) The Author(s) 2021

\begin{abstract}
This short note has to make alive the overlooked work of Pedrick. It is rather a tour guide than exhausted examination of the content and intends to serve potential explorers of diverse kinds of reproducing kernel (Hilbert) spaces, the topic mushrooming nowadays.
\end{abstract}

\section{Some Extracts from Aronszajn: Updated}

Let $X$ be an arbitrary set, refer to it as to underlying set; the function $K: X \times X \rightarrow \mathbb{C}$ is called a kernel on $X$. Let $\mathcal{H}$ be a Hilbert space of complex valued functions on $X$. We say that the couple $(K, \mathcal{H})$ has the reproducing property ${ }^{1}$ if for the sections $K_{x}$ of the kernel $K$

$$
K_{x} \stackrel{\text { def }}{=} K(\cdot, x) \text { belongs to } \mathcal{H} \text { for every } x \in X,
$$

and $^{2}$

$$
f(x)=\left\langle f, K_{x}\right\rangle, \quad f \in \mathcal{H}, x \in X .
$$

\footnotetext{
1 We maintain the commonly used term reproducing kernel Hilbert space, RKHS briefly, despite linguistic nuances which may not emphasise enough that both members of the couple have equal rights.

2 The functions $K_{x}$ may be called kernel functions if necessary, as they are functions of a single variable and come from the kernel $K$, a function of two variables like $K$ in Analysis is generally called a kernel; some authors seem not to be aware of this subtle distinction.

3 We spread the notation "lin" for the linear span while "clolin" for the closed linear one.
}

To Henk with appreciation.

Communicated by Seppo Hassi.

This article is part of the topical collection "Recent Developments in Operator Theory - Contributions in Honor of H.S.V. de Snoo" edited by Jussi Behrndt and Seppo Hassi.

$凶 \quad$ Franciszek Hugon Szafraniec umszafra@cyf-kr.edu.pl

1 Instytut Matematyki, Uniwersytet Jagielloński, ul. Łojasiewicza 6, 30348 Kraków, Poland 
The following properties ${ }^{3}$ are crucial and of perspective usefulness.

$1^{\mathrm{o}} \mathcal{D}_{K} \stackrel{\text { def }}{=} \operatorname{lin}\left\{K_{x}: x \in X\right\}$ is dense in $\mathcal{H}$.

$2^{\mathrm{o}}$ The kernel $K$ is positive definite (PD in short), that is

$$
\sum_{i, j=1}^{N} K\left(x_{i}, x_{j}\right) \lambda_{i} \bar{\lambda}_{j} \geq 0, \quad x_{1}, \ldots, x_{N} \in X, \lambda_{1}, \ldots \lambda_{N} \in \mathbb{C}
$$

which consequently forces Hermitian symmetry

$$
K(x, y)=\overline{K(y, x)}, \quad x, y \in X
$$

as well as the Schwarz inequality

$$
\begin{gathered}
\left|\sum_{i, k=1}^{M, N} K\left(x_{i}, y_{k}\right) \lambda_{i} \bar{\mu}_{k}\right|^{2} \leq \sum_{i, j=1}^{M} K\left(x_{i}, x_{j}\right) \lambda_{i} \bar{\lambda}_{j} \sum_{k, l=1}^{N} K\left(y_{k}, y_{l}\right) \mu_{k} \bar{\mu}_{l}, \\
x_{1}, \ldots, x_{M}, y_{1}, \ldots, y_{N} \in X, \lambda_{1}, \ldots \lambda_{M}, \mu_{1}, \ldots, \mu_{N} \in \mathbb{C} .
\end{gathered}
$$

$3^{\circ}$ The evaluation functionals

$$
\Phi_{x}: f \ni \mathcal{H} \mapsto f(x) \in \mathbb{C}
$$

are continuous for every $x \in X$; this is an immediate consequence of (2).

$4^{\mathrm{o}}$ The reproducing formula (2) gives at once

$$
K(x, y)=\left\langle K_{y}, K_{x}\right\rangle, \quad x, y \in X
$$

This is a powerful formula because it is equivalent in a sense to the reproducing property.

$5^{\circ}$ The practical formula which goes back to Zaremba (see $[15$, p. 170]) is

$$
K(x, y)=\sum_{\alpha \in \mathrm{A}} \phi_{\alpha}(x) \overline{\phi_{\alpha}(y)}, \quad x, y \in X
$$

provided the right hand side converges (which may be understood as an integral if $\mathrm{A}$ is uncountable).

$6^{\circ}$ A complex function $f$ on $X$ is in $\mathcal{H}$ if and only if there is a constant $C>0$ such that

$$
\begin{gathered}
\left|\sum_{i=1}^{N} f\left(x_{i}\right) \lambda_{i}\right|^{2} \leq C \sum_{i, j=1}^{N} K\left(x_{i}, x_{j}\right) \lambda_{i} \bar{\lambda}_{j}, \\
x_{1}, \ldots, x_{N} \in X, \lambda_{1}, \ldots \lambda_{N} \in \mathbb{C}
\end{gathered}
$$

in such a situation $\|f\|_{\mathcal{H}}$ is the smallest $C$, for which the formula (8) holds. 
This is a handy RKHS test identifying functions on $X$ which constitute the space $\mathcal{H}$.

Let us memorise any of the two members of a reproducing couple determines (uniquely) the other, cf. footnote ${ }^{1}$.

\section{Kernel $K \Longrightarrow$ Space $\mathcal{H}$}

Define $\mathcal{D}_{K}$ as in $1^{\circ}$ for a given a PD kernel $K$ on $X$. Reading backwards the property (6) leads to the definition

$$
\left\langle\sum_{i=1}^{M} \lambda_{i} K_{x_{i}}, \sum_{k=1}^{N} \mu_{k} K_{y_{k}}\right\rangle \stackrel{\text { def }}{=} \sum_{i, k=1}^{M, N} K\left(x_{i}, y_{k}\right) \lambda_{i} \bar{\mu}_{k}
$$

A direct argument convinces us that the function $\langle\cdot,-\rangle: \mathcal{D}_{K} \times \mathcal{D}_{K} \rightarrow \mathbb{C}$ is well defined and linear in the first variable; the Hermitian symmetry (4) makes it antilinear in the second variable and well defined at large. Positive definiteness (3) of $K$ ensures the aforesaid function to be an semi-inner product. Definition (9) incorporates the reproducing property (2) satisfied now on $\mathcal{D}_{K}$ and this is enough to conclude that the semi-inner product in question becomes an inner product.

Now we can execute the Hilbert space $\mathcal{H}$ with simple (pointwise) completion according to the lemma below.

Lemma 1 For a unitary space $\mathcal{D}$ of complex functions on $X$ the following conditions are equivalent:

- there exists (necessarily, exactly one) Hilbert space $\mathcal{H}$ of functions on $X$ with reproducing kernel $K$ on $X$ such that $\mathcal{D}$ is dense in $\mathcal{H}$;

- the space $\mathcal{D}$ has reproducing kernel $K$ such that $\mathcal{D}_{K}$ is dense in $\mathcal{D}$;

- the space $\mathcal{D}$ has two properties:

- the evaluation functionals ${ }^{4}$

$$
\Phi_{x}: f \ni \mathcal{D} \mapsto f(x) \in \mathbb{C}, \quad x \in X,
$$

are continuous,

- for each sequence $\left(f_{n}\right)_{n=0}^{\infty}$, which is Cauchy in $\mathcal{D}$, convergence of $f_{n}(x) \rightarrow 0$ for all $x \in X$ implies $\left\|f_{n}\right\| \rightarrow 0$.

\section{Kernel $K \Longleftarrow$ Space $\mathcal{H}$}

This way is fast. Suppose a Hilbert space $\mathcal{H}$ of complex functions on $X$ is granted with the property $3^{\circ}$. Then immediately the Riesz representation theorem for the functionals $\Phi_{x}$ determines the kernel functions $K_{x}$ and recurring the formula (6) ends up the whole story.

\footnotetext{
4 Notice now, unlike (5) they are defined on an inner product space exclusively.
} 
Let us notice the strength of $3^{\circ}$ is usually caused to happen due to some influential source out of the space $\mathcal{H}$.

Another way to define the kernel is to use Zaremba's formula (7) starting from a given family $\left\{\varphi_{\alpha}\right\}$ of functions in $\mathcal{H}$ which is total. Then the definition of the kernel corresponding to $\mathcal{H}$ is immediate ${ }^{5}$.

The basic reference is [1]. As for the further details concerning this part consult [8]. The monograph [12] would be for the benefit of those who are forbearing; it is in fact the very extension of [8]. If someone is desperate for having a look at English version of some parts of [8] the surveys $[10,11]$ may serve for this in a way.

\section{Pedrick's Reinforcement of Aronszajn's Scheme}

George Pedrick, Aronszajn's PhD student, in his 1959 dissertation [5] extended the concept of reproducing kernel Hilbert spaces much beyond the customary context of scalar valued functions and kernels. Even so his ideology was to make the two recipes equivalent. We pick up here the main points of [5] adjusting them to contemporary developments and needs rather than to scrutinise his treatise. This means Pedrick's main idea itself is implemented here in a rather effortless way rectifying some meanders which appear in [5]. Active engagement of the reader may be called for.

Given a set $X$ of arbitrary nature, let $\mathcal{E}$ be a locally convex space and let $\mathcal{E}^{\prime}$ stand for its topological anti-dual. ${ }^{6}$ This choice is a kind of sample rather, other dualities based on $\mathcal{E}$ can be taken into account as well.

The following two items are the main objects in [5, cf. p.16]

- a complex Hilbert space $\mathcal{G} \subset \mathcal{E}^{X}$ which is composed of $\mathcal{E}$-valued functions on $X$,

- a family of linear operators $K(x, y): \mathcal{E}^{\prime} \rightarrow \mathcal{E}, x, y \in X$, which may be condensed as $^{7} K: X \times X \rightarrow \boldsymbol{L}\left(\mathcal{E}^{\prime}, \mathcal{E}\right)$ and which in turn let $K$ be thought of as $\boldsymbol{L}\left(\mathcal{E}^{\prime}, \mathcal{E}\right)$ valued kernel on $X$.

Think of $(K, \mathcal{G})$ as the reproducing kernel Hilbert space couple on $X$ if

$$
\text { the functions } K_{x, e^{\prime}} \stackrel{\text { def }}{=} K(\cdot, x) e^{\prime} \text { are in } \mathcal{G} \text { for any } x \in X, e^{\prime} \in \mathcal{E}^{\prime}
$$

$\underline{\text { and }}$

$$
\left\langle\varphi(x), e^{\prime}\right\rangle_{\left(\mathcal{E}, \mathcal{E}^{\prime}\right)}=\left\langle\varphi, K_{x, e^{\prime}}\right\rangle_{\mathcal{G}}, \quad \varphi \in \mathcal{G}, x \in X, e^{\prime} \in \mathcal{E}^{\prime}
$$

where the subscript $\left(\mathcal{E}, \mathcal{E}^{\prime}\right)$ fixes "anti-duality" between the spaces $\mathcal{E}$ and $\mathcal{E}^{\prime}$ (the latter is anti-linear with respect to the second variable), and $\langle\cdot,-\rangle_{\mathcal{G}}$ does the inner product of the Hilbert space $\mathcal{G}$. Notice the formula (12) is just the reproducing property as adjusted to the current circumstances.

\footnotetext{
5 Notice that it needs not to be orthonormal, this comes out a posteriori as an extra condition required, cf. [8].

6 This is the space of all anti-linear(=linear conjugate) continuous functionals on $\mathcal{E}$, which just coincides with the space of all complex conjugates of continuous linear functionals on $\mathcal{E}$, the members of the topological dual of $\mathcal{E}$. For duality consult Sect. 5 .

${ }^{7} \boldsymbol{L}(\mathcal{X}, \mathcal{Y})$ denotes the totality of all linear operators from $\mathcal{X}$ into $\mathcal{Y}$, which by the way is a complex linear space; as always shorten $L(\mathcal{X}, \mathcal{X})$ to $L(\mathcal{X})$.
} 
Due to the fact that $\mathcal{E}^{\prime}$ separates the points of $\mathcal{E}$ the reproducing formula (12) forces the set

$$
\mathcal{D} \stackrel{\text { def }}{=} \operatorname{lin}\left\{K_{x, e^{\prime}}: x \in X, e^{\prime} \in \mathcal{E}^{\prime}\right\}
$$

to be dense in $\mathcal{G}$.

Positive definiteness reads as follows

$$
\sum_{i, j=1}^{N}\left\langle K\left(x_{i}, x_{j}\right) e_{i}^{\prime}, e_{j}^{\prime}\right\rangle_{\left(\mathcal{E}, \mathcal{E}^{\prime}\right)} \geq 0, \quad x_{1}, \ldots, x_{N} \in X, e_{1}^{\prime}, \ldots e_{N}^{\prime} \in \mathcal{E}^{\prime}
$$

Like in the scalar case the reproducing formula (12) concludes the evaluation "functional" (or rather the evaluation operator in the current setting)

$$
\Phi_{x}: \varphi \ni \mathcal{G} \mapsto \varphi(x) \in \mathcal{E}, \quad x \in X
$$

is $\left(\mathcal{G},\langle\cdot,-\rangle_{\mathcal{G}}\right)$ to $\left(\mathcal{E}, \sigma\left(\mathcal{E}, \mathcal{E}^{\prime}\right)\right)$ continuous.

Another consequence of the reproducing formula (12) is

$$
\left\langle K(\cdot, x) e^{\prime}, K(\cdot, y) f^{\prime}\right\rangle_{\mathcal{G}}=\left\langle K(y, x) e^{\prime}, f^{\prime}\right\rangle_{\left(\mathcal{E}, \mathcal{E}^{\prime}\right)}
$$

a counterpart of (6).

Zaremba's formula (7) as in $5^{\circ}$ has its meaning in this setting too.

The criterion $6^{\circ}$ (RKHS test) under the present circumstances takes the form.

$7^{\circ}$ A function $\varphi \in \mathcal{E}^{X}$ is in $\mathcal{G}$ if and only if there is a constant $C>0$ such that

$$
\begin{aligned}
& \left|\sum_{i=1}^{N}\left\langle\varphi\left(x_{i}\right), e_{i}^{\prime}\right\rangle_{\left(\mathcal{E}, \mathcal{E}^{\prime}\right)}\right|^{2} \leq C \sum_{i, j=1}^{N}\left\langle K\left(x_{i}, x_{j}\right) e_{i}^{\prime}, e_{j}^{\prime}\right\rangle_{\left(\mathcal{E}, \mathcal{E}^{\prime}\right)}, \\
& x_{1}, \ldots, x_{N} \in X, e_{1}, \ldots e_{N} \in \mathbb{C}
\end{aligned}
$$

Consequently $\|\varphi\|_{\mathcal{G}}$ is the smallest $C$ for which the formula (17) holds.

Because the RKHS test plays an important role in the next section we say a couple of words about its proof. The reproducing formula (12) makes the "only if" part of $7^{\circ}$. For the other part, (17) makes it possible to apply the Riesz representation to the functional (well defined on the whole of $\mathcal{G}$ indeed)

$$
\begin{aligned}
& \sum_{i=1}^{N} K\left(\cdot, x_{i}\right) e_{i}^{\prime} \lambda_{i} \mapsto \sum_{i=1}^{N}\left\langle\varphi\left(x_{i}\right), e_{i}^{\prime}\right\rangle_{\left(\mathcal{E}, \mathcal{E}^{\prime}\right)} \lambda_{i}, \\
& x_{1}, \ldots, x_{N} \in X, e_{1}^{\prime}, \ldots, e_{N}^{\prime} \in \mathcal{E}^{\prime}, \lambda_{1} \ldots \lambda_{N} \in \mathbb{C} .
\end{aligned}
$$

Then use (12) and again the fact that $\mathcal{E}^{\prime}$ separates the points of $\mathcal{E}$, to identify $\varphi$ as a member of $\mathcal{G}$.

Needless to say the above arguments work well also for $6^{\circ}$. 
The very bottom condition in Lemma 1 which is of rather delicate matter is explained in $\$ 5$ of [5]. We deliberately glance through this section because our decisive point is in what appears in the following one.

\section{Pedrick's "Tilde Correspondence"}

This is what definitely has to be brought out of Pedrick's thesis and put forward. He suggests to go back from the vector valued case of Sect. 2 to that of scalar valued as in Sect. 1 by a simple, and as it turns out, very efficient device (cf. [5, §2, p.22]); we arrange it in a slightly modified way just to make it fit in with the setup of section 1 .

With notations of Sect. 2 we offer the following definitions

$$
\widetilde{K}\left(x, e^{\prime}, y, f^{\prime}\right) \stackrel{\text { def }}{=}\left\langle K(x, y) e^{\prime}, f^{\prime}\right\rangle_{\left(\mathcal{E}, \mathcal{E}^{\prime}\right)}, \quad x, y \in X, e^{\prime}, f^{\prime} \in \mathcal{E}^{\prime}
$$

and for $\varphi \in \mathcal{E}^{X}$ encode

$$
\begin{gathered}
\tilde{\varphi}\left(x, e^{\prime}\right) \stackrel{\text { def }}{=}\left\langle\varphi(x), e^{\prime}\right\rangle_{\left(\mathcal{E}, \mathcal{E}^{\prime}\right)}, \quad\left(x, e^{\prime}\right) \in \widetilde{X} \stackrel{\text { def }}{=} X \times \mathcal{E}^{\prime} ; \\
\tilde{\mathcal{G}} \stackrel{\text { def }}{=}\{\tilde{\varphi}: \varphi \in \mathcal{G} \text { and } \tilde{\varphi} \text { defined in (19) }\} .
\end{gathered}
$$

Remark 2 Now the deal is with complex valued kernel $\widetilde{K}$. Because $\widetilde{K}$ is linear in the second variable and antilinear in the fourth, formula (14) ensures its positive definiteness in the sense of (3) having now the form

$$
\sum_{i, j=1}^{N} \widetilde{K}\left(x_{i}, e_{i}^{\prime}, x_{j} e_{j}^{\prime}\right) \geq 0, \quad x_{1}, \ldots, x_{N} \in X, e_{1}^{\prime}, \ldots e_{N}^{\prime} \in \mathcal{E}^{\prime}
$$

Therefore $\widetilde{K}$ has its reproducing kernel Hilbert space $\tilde{\mathcal{H}}$ composed of complex functions on $\widetilde{X} \stackrel{\text { def }}{=} X \times \mathcal{E}^{\prime}$.

If there is a need to consider other duality, like $(\mathcal{E}, \mathcal{F})$ exposed in Sect. 5, then the topological one $\left(\mathcal{E}, \mathcal{E}^{\prime}\right)$ an automatic "copy-paste" solves the problem globally.

Formula (18) allows us to create the kernel functions $\widetilde{K}_{y, f^{\prime}}$ as

$$
\widetilde{K}_{y, f^{\prime}} \stackrel{\text { def }}{=} \widetilde{K}\left(\cdot,-, y, f^{\prime}\right) \quad y, f^{\prime} \in X \times \mathcal{E}^{\prime}
$$

cf. footnote ${ }^{2}$.

Due to the fact that $\mathcal{E}^{\prime}$ separates the points of $\mathcal{E}$ the mapping in (19) as defined on $\mathcal{E}^{X}$ is injective. Even more there is a simple relationship ${ }^{8}$ between the topologies of $\mathcal{G}$ and $\widetilde{\mathcal{G}}$.

Even more, the main goal is to check whether $\widetilde{\mathcal{G}}$ is just the RKHS partner of $\widetilde{K}$.

\footnotetext{
8 Pedrick declares these topologies to coincide a priori, we prefer to conclude this going another way around, see Proposition 3.
} 
Theorem 3 For $\varphi \in \mathcal{G}$

$$
\|\varphi\|_{\mathcal{G}}=\|\tilde{\varphi}\|_{\tilde{\mathcal{G}}}
$$

where $\|\cdot\|_{\widetilde{\mathcal{G}}}$ stands for the norm in $\widetilde{\mathcal{G}}$. Consequently, $(\widetilde{K}, \widetilde{\mathcal{G}})$ is a reproducing couple.

Theorem 3 and everything around are the quintessence of Pedrick's considerations.

Proof The link established in (18) and (19) yields

$$
\begin{aligned}
& \left|\sum_{i=1}^{N}\left\langle\varphi\left(x_{i}\right), e_{i}^{\prime}\right\rangle_{\left(\mathcal{E}, \mathcal{E}^{\prime}\right)} \lambda_{i}\right|\left(\sum_{i, j=1}^{N}\left\langle K\left(x_{i}, x_{j}\right) e_{i}^{\prime}, e_{j}^{\prime}\right\rangle_{\left(\mathcal{E}, \mathcal{E}^{\prime}\right)} \lambda_{i} \bar{\lambda}_{j}\right)^{-1 / 2} \\
& =\left|\sum_{i=1}^{N} \tilde{\varphi}\left(x_{i}, e_{i}^{\prime}\right) \lambda_{i}\right|\left(\sum_{i, j=1}^{N} \widetilde{K}\left(x_{i}, e_{i}^{\prime}, x_{j}, e_{j}^{\prime}\right) \lambda_{i} \bar{\lambda}_{j}\right)^{-1 / 2}
\end{aligned}
$$

Carrying out "sup" over

$$
x_{1}, \ldots, x_{N} \in X, e_{1}^{\prime}, \ldots e_{N}^{\prime} \in \mathcal{E}^{\prime}, \lambda_{1}, \ldots \lambda_{N} \in \mathbb{C}
$$

in both sides of the above equality, invoking $6^{\circ}$ and $7^{\circ}$ we arrive at (23). Now (23) applied to the right hand side of (12) and the defining formula (19) to its left hand side leads to the reproducing formula for $\widetilde{K}$ with $\widetilde{\mathcal{G}}$ as its Hilbert space.

\section{Some Upcoming Cases}

Due to Theorem 3 the mapping

$$
\mathcal{G} \ni \varphi \mapsto \tilde{\varphi} \in \widetilde{\mathcal{G}}
$$

besides being a unitary operator between two Hilbert spaces $\mathcal{G}$ and $\widetilde{\mathcal{G}}$ of functions preserves the reproducing property of both couples $(K, \mathcal{G})$ and $(\widetilde{K}, \widetilde{\mathcal{G}})$. Thus we may think of RKHS isomorphism in all respects.

Looking now at (18) as an equality between $\widetilde{K}$ and $K$ one may catch an idea of going along it in any the two of directions: from operator (vector) valued kernels to scalar ones and the other way. "The other way" seems to be more interesting to monitor, let us do this roughly leaving the details for the reader. Anyway it relies on Proposition 12, which may be helpful.

Remark 4 Notice that the underlying set for the kernel $\widetilde{K}$ is $X \times \mathcal{E}$ amalgamating two factors $X$ and $\mathcal{E}$ of different (usually independent) structure. The set $X$ is often of algebraic nature while $\mathcal{E}$ bears a geometrical structure,

\section{Particularising $X$ and $\mathcal{E}$}

The set $X$ can be provided with some algebraic structure, the most notable the structure of semigroup (or, a bit more generally, a semigroup of actions on $X$ ); we think 
of the semigroup acting multiplicatively unless it is commutative when the additive notation is customarily in use. Existence of unit in a semigroup is not a common assumption however for the time being we assume that. We also enrich the structure of $X$ introducing an involution in it which gives the term *-semigroup, consult [12, Chapter 2].

Keep the space $\mathcal{E}$ normed. If $\widetilde{K}(1, f, 1, f)=\|f\|^{2}$ we call $\widetilde{K}$ isometric. The embedding

$$
V: \mathcal{E}^{\prime} \ni f^{\prime} \rightarrow \widetilde{K}_{1, f^{\prime}} \in \tilde{\mathcal{H}}
$$

in case of isometric $\widetilde{K}$ is just an isometry.

Let $\widetilde{K}_{y, f^{\prime}}$ be the kernel functions (22) generated by the scalar kernels $\widetilde{K}$, cf. (1). Under the current circumstances we can consider the following operators.

\section{The Operators}

The semigroup structure of $X$ allows us to introduce two linear operators in $\tilde{\mathcal{H}}$ playing a basic role in dilation theory. Take $u \in X$ and define the mapping

$$
\mathbb{C}^{X \times \mathcal{E}} \ni F \mapsto F_{u} \in \mathbb{C}^{X \times \mathcal{E}}
$$

with

$$
F_{u}(s, f) \stackrel{\text { def }}{=} F(u s, f), \quad(s, f) \in X \times \mathcal{E},
$$

and the linear space

$$
\mathcal{D}(u) \stackrel{\text { def }}{=}\left\{F \in \mathcal{H}: F_{u} \in \widetilde{\mathcal{H}}\right\} .
$$

Now we will try to define two linear operators in $\tilde{\mathcal{H}}$ corresponding to $u \in X$. First of them, $\Phi(u)$, can be set definitely right now

$$
\mathcal{D}(\Phi(u)) \stackrel{\text { def }}{=} \mathcal{D}(u), \quad \Phi(u) F \stackrel{\text { def }}{=} F_{u}, \quad F \in \mathcal{D}(u) .
$$

The second, $\Phi_{u}$, relies on

$$
\Phi_{u} \widetilde{K}_{s, f} \stackrel{\text { def }}{=} \widetilde{K}_{u s, f}, \quad s \in X, f \subset \mathcal{E}
$$

and requires some caution, it extends linearly to a well-defined linear operator on $\mathcal{D}\left(\Phi_{u}\right) \stackrel{\text { def }}{=} \mathcal{D}_{\widetilde{K}}$ if and only if

$$
\sum_{i} \xi_{i} \widetilde{K}_{s_{i}, f_{i}}=0 \Longrightarrow \sum_{i} \xi_{i} \widetilde{K}_{u s_{i}, f_{i}}=0,
$$

otherwise it is a linear relation. Regardless $\Phi_{u}$ is already a well defined operator or not the formula

$$
\Phi_{u} V f=K_{u, f}
$$

holds true, cf. (25), provided $X$ is unital. 
Proposition 5 The basic link is

$$
\left\langle\Phi(u) F, K_{x, f^{\prime}}\right\rangle=\left\langle F, \Phi_{u} K_{x, f^{\prime}}\right\rangle, \quad F \in \mathcal{D}(u),\left(x, f^{\prime}\right) \in X \times \mathcal{E}^{\prime}
$$

Corollary 6 The following consequences of Proposition 5 are important to be notified.

1 The operator $\Phi(u)$ is closed.

2 If $\Phi(u)$ is densely defined, that is $\mathcal{D}(u)$ is dense in $\tilde{\mathcal{H}}$, then the operator $\Phi_{u}$ is well defined on $\mathcal{D}$.

3 If $\Phi(u)$ is densely defined then

$$
\Phi_{u}^{*}=\Phi(u), \Phi_{u} \text { is closable and } \bar{\Phi}_{u}=\Phi(u)^{*} .
$$

Boundedness of $\Phi(u)$ and $\Phi_{u}$ is a subject of separate analysis. In the case of $*-$ involution semigroups there is a bunch of equivalent conditions to $(33)$, cf. [7,9].

Proposition $7 \quad \Phi_{u}$ is a well-defined bounded operator if and only if there exists $c(u) \geqslant$ 0 such that

$$
\begin{aligned}
& \sum_{i, j=1}^{N} \widetilde{K}\left(u s_{i}, f_{i}, u s_{j}, f_{j}\right) \leq c(u) \sum_{i, j=1}^{N} \widetilde{K}\left(s_{i}, f_{i}, s_{j}, f_{j}\right) \\
& s_{1}, \ldots, s_{N} \in X, f_{1}, \ldots f_{N} \in \mathcal{E}
\end{aligned}
$$

If this happens $\Phi(u)$ is a densely defined operator, and both $\Phi_{u}$ and $\Phi(u)$ bounded operators with

$$
\left\|\Phi_{u}\right\|=\|\Phi(u)\| \leq c(u) .
$$

Let us also have a look at algebraic properties of the mappings $u \mapsto \Phi(u)$ and $u \mapsto \Phi_{u}$.

Proposition 8 Suppose that for each $u \in X(29)$ holds. Then $\mathcal{D}\left(\Phi_{u}\right)$ is invariant on $\Phi_{u}$, that is, $\Phi_{u} \mathcal{D}\left(\Phi_{u}\right) \subset \mathcal{D}\left(\Phi_{u}\right)$, and the mapping $u \mapsto \Phi_{u}$ is multiplicative, that is

$$
\Phi_{u v} F=\Phi_{u} \Phi_{v} F, \quad u, v \in X, \quad F \in \mathcal{D}_{K}
$$

The mapping $u \mapsto \Phi(u)$ is anti-multiplicative, which here means that

$$
\Phi(u) \Phi(v) F=\Phi(v u) F, \quad u, v \in X, \quad F \in \mathcal{D}(\Phi(u) \Phi(v)) .
$$

Since we have all the ingredients ready, it is time for the first and most general dilation theorem.

Theorem 9 Let $X$ be a unital semigroup, $\mathcal{E}$ a normed space, and $K$ a positive definite isometric kernel on $X \times \mathcal{E}$. Then, in the RHKS $\widetilde{\mathcal{H}}$ corresponding to $\widetilde{K}$ we have formulae ${ }^{9}$

$$
\widetilde{\mathcal{H}}=\operatorname{clolin}\left\{\Phi_{u} V f: u \in X, f \in \mathcal{E}\right\}=\operatorname{clolin}\left\{\widetilde{K}_{u, f}: u \in X, f \in \mathcal{E}\right\}
$$

\footnotetext{
9 Note that first of these conditions could be omitted without any loss, since it is a fact contained in the definition of the reproducing kernel Hilbert space.
} 


$$
\widetilde{K}(s, f, t, g)=\left\langle\Phi_{t} V g, \Phi_{s} V f\right\rangle=\left\langle\widetilde{K}_{t, g}, \widetilde{K}_{s, f}\right\rangle, \quad(s, f),(t, g) \in X \times \mathcal{E},
$$

where all the objects mentioned in the conclusion have been defined already.

Remark 10 The remarkable attraction of Pedrick's "tilde correspondence" is that the resulting reproducing kernel Hilbert space composed of complex valued (scalar) functions can be decoded using the RKHS test $6^{\circ}$, p. 2. In particular it can help localising the domains of operators appearing in Corollary 6 as is done in [13] in order to detect subnormality of unbounded operators.

\section{*-Invariance}

Suppose $X$ is a $*$-semigroup with unit 1 and $K$, therefor $\widetilde{K}$ is invariant in the sense

$$
\begin{aligned}
& \left\langle K(u x, y) e^{\prime}, f^{\prime}\right\rangle_{\left(\mathcal{E}, \mathcal{E}^{\prime}\right)}=\left\langle K\left(x, u^{*} y\right) e^{\prime}, f^{\prime}\right\rangle_{\left(\mathcal{E}, \mathcal{E}^{\prime}\right)} \\
& K\left(u x, f^{\prime}, y, g^{\prime}\right)=K\left(x, f^{\prime}, u^{*} y, g^{\prime}\right), \\
& u, s, t \in X, f^{\prime}, g^{\prime} \in \mathcal{E}^{\prime} .
\end{aligned}
$$

This introduces a new definition

$$
\omega\left(t^{*} s, f^{\prime}, g^{\prime}\right) \stackrel{\text { def }}{=} K\left(s, f^{\prime}, t, g^{\prime}\right)
$$

which reduces the number of variables by one; call $\omega$ a form.

In the fundamental paper of Sz.-Nagy [14], which unfortunately is disregarded by the so called experts, forms generated by bounded operators is considered in "théorème principal" and dilation results stated by them. Among the forms those determined by contractions (here the famous Sz.-Nagy 1953 dilation theorem is materialised), semispectral (POV) measures, operator moment problems on bounded intervals are included as special cases. This is presented in details though in a rather general setup in [9].

\section{Spaces in Duality: Repository}

Remark 11 Suppose $\mathcal{H}$ is a complex Hilbert space and $\Phi$ is a continuous linear functional on $\mathcal{H}$. Then there is a unique element $g_{\Phi}$ of $\mathcal{H}$ such that

$$
\Phi(f)=\left\langle f, g_{\Phi}\right\rangle_{\mathcal{H}}, \quad f \in \mathcal{H}
$$

This is the classical F. Riesz representation theorem; the correspondence $\Phi \longleftrightarrow g_{\Phi}$ is anti-linear.

Duality as it appears in Functional Analysis, cf. [3, Chapter 3], [2, p. 155], [4, p. 59] declares three objects to be given: linear spaces $\mathcal{E}$ and $\mathcal{F}$, and a bilinear form

$$
B: \mathcal{E} \times \mathcal{F} \ni(f, g) \mapsto B(x, y) \in \mathbb{C}
$$


which is separating in a sense that

$$
\begin{aligned}
& B(f, g)=0 \text { for all } g \in \mathcal{F} \Longrightarrow f=0 \\
& B(f, g)=0 \text { for all } f \in \mathcal{E} \Longrightarrow g=0 .
\end{aligned}
$$

The spaces $\mathcal{E}$ and $\mathcal{F}$ when accompanied by $B$ are referred to as being in duality. The most recognised examples are

- $\mathcal{E}$ is a linear space and $\mathcal{F}=\mathcal{E}^{*}$, where $\mathcal{E}^{*}$ is the algebraic dual of $\mathcal{E}$, that is the space of all linear functionals on $\mathcal{E}$;

- $\mathcal{E}$ is a locally convex space and $\mathcal{F}=\mathcal{E}^{\prime}$, where $\mathcal{E}^{\prime}$ is the topological dual of $\mathcal{E}$, that is the space of all continuous linear functionals on $\mathcal{E}$

the bilinear form in both these cases is just the standard pairing, that is $B=\langle\cdot,-\rangle$; from now on we use the latter instead of $B$.

The first of these two cases can always be directed in the second by introducing the so called $\sigma(\mathcal{E}, \mathcal{F})$ topology in $\mathcal{E}$ or reversing the role the spaces play a $\sigma(\mathcal{F}, \mathcal{E})$ topology in $\mathcal{F}$; in general they are not the only one possibilities. More precisely, if $\mathcal{E}$ and $\mathcal{F}$ are arbitrary linear spaces being in duality furnished with the separating bilinear form $B=\langle\cdot,-\rangle$ there is always a topology on $\mathcal{E}$, just $\sigma(\mathcal{E}, \mathcal{F})$, making it a locally convex space and turning $\mathcal{F}$ into its topological dual; the same can applied by reversing the role of spaces. We use the customarily notation $\sigma(\mathcal{E}, \mathcal{F})$ and $\sigma(\mathcal{F}, \mathcal{E})$ for these topology while for the bilinear form we use the subscript $(\mathcal{E}, \mathcal{F})$ or $(\mathcal{F}, \mathcal{E})$ to indicate the proper choice, see [2, Definition (3.84), p 156].

Important change. Because the Riesz representation theorem identifies the topological dual of a Hilbert space with the space itself establishing merely an antilinear mapping we prefer to make a change introducing a prefix "anti" to tailor the aforesaid duality to the newly required situation; no essential technical modification is needed $^{10}$ to implement this. In practice we start from the duality form $B=\langle\cdot,-\rangle$ to be Hermitian bilinear, that is linear in the first variable, anti-linear (aka Hermitian linear or even sometimes called linear conjugate) in the second. This approach is supported by Remark 11 .

Here is an excerpt from [2, Theorem (38.7)] in its (antilinear) modified form.

Proposition 12 A linear functional $F$ on $\mathcal{E}$ is $\sigma(\mathcal{E}, \mathcal{F})$ continuous if and only if there is (necessarily a unique) $g \in \mathcal{F}$ such that $F=\langle\cdot, g\rangle_{(\mathcal{E}, \mathcal{F})}$. The dual statement holds as well and says an anti-linear functional $G$ on $\mathcal{F}$ is $\sigma(\mathcal{F}, \mathcal{E})$ continuous if and only if there is $f \in \mathcal{E}$ such that $G=\langle f, \cdot\rangle_{(\mathcal{E}, \mathcal{F})}$.

For some support in this matter look at $[5, \S 1]$ or [6], both are given the same flavour.

Open Access This article is licensed under a Creative Commons Attribution 4.0 International License, which permits use, sharing, adaptation, distribution and reproduction in any medium or format, as long as you give appropriate credit to the original author(s) and the source, provide a link to the Creative Commons licence,

10 This is because anti-linear(=linear conjugate) functionals on $\mathcal{E}$ coincide with complex conjugates of linear functionals on $\mathcal{E}$. 
and indicate if changes were made. The images or other third party material in this article are included in the article's Creative Commons licence, unless indicated otherwise in a credit line to the material. If material is not included in the article's Creative Commons licence and your intended use is not permitted by statutory regulation or exceeds the permitted use, you will need to obtain permission directly from the copyright holder. To view a copy of this licence, visit http://creativecommons.org/licenses/by/4.0/.

\section{References}

1. Aronszajn, N.: Theory of reproducing kernels. Trans. Am. Math. Soc. 68, 337-404 (1950)

2. Berberian, S.K.: Lectures in Functional Analysis and Operator Theory. Springer, New York (1974)

3. Horváth, J.: Topological Vector Spaces and Distributions, vol. I. Addison-Wesley Publishing Co, Reading (1966)

4. Pedersen, G.K.: Analysis Now. Springer, New York (1989)

5. Pedrick, G.: Theory of reproducing kernels for Hilbert spaces of vector valued functions, Thesis (Ph.D.)-University of Kansas, pp. 59 (1958)

6. Schwartz, L.: Sous-espaces hilbertiens d'espaces vectoriels topologiques et noyaux associés (noyaux reproduisants). J. Analyse Math. 13, 115-256 (1964)

7. Szafraniec, F.H.: Boundedness of the shift operator related to positive definite forms: an application to moment problems. Ark. Mat. 19, 251-259 (1981)

8. Szafraniec, F.H.: Przestrzenie Hilberta $\mathrm{z}$ jądrem reprodukującym (Positive definite kernels and Hilbert $C *$-modules, Reproducing kernel Hilbert spaces, in Polish). Wydawnictwo Uniwersytetu Jagiellońskiego, Kraków (2004)

9. Szafraniec, F.H.: Murphy's Positive definite kernels and Hilbert $C^{\prime}$-modules reorganized. Banach Center Public. 89, 275-295 (2010)

10. Szafraniec, F.H.: The reproducing kernel property and its space: the basics. In: Alpay, Daniel (ed.) Operator Theory, vol. 1, pp. 3-30. Springer, Basel (2015)

11. Szafraniec, F.H.: The reproducing kernel property and its space: more or less standard examples of applications. In: Alpay, Daniel (ed.) Operator Theory, vol. 1, pp. 31-58. Springer, Basel (2015)

12. Szafraniec, F.H.: The Reproducing Property: Spaces and Operators. Cambridge University Press (in progress)

13. Szafraniec, F.H.: The Sz.-Nagy "théorème principal" extended. Application to subnormality. Acta Sci. Math. (Szeged) 57, 249-262 (1993)

14. Sz.-Nagy, B.: Prolongements des transformations de l'espace de Hilbert qui sortent de cet espace. Appendice au livre "Leçons d'analyse fonctionnelle" par F. Riesz et B. Sz.-Nagy, Akadémiai Kiadó, Budapest (1955)

15. Zaremba, S.: L'équation biharmonique et une classe remarquable de functions fondamentales harmoniques. Bull. Int. l'Acad. Sci. Cracovie 147-196 (1907)

Publisher's Note Springer Nature remains neutral with regard to jurisdictional claims in published maps and institutional affiliations. 